\title{
Multicomponent comprehensive care was as effective as usual care for decreasing major bleeding in older patients on warfarin
}

\author{
Beyth RJ, Quinn L, Landefeld CS. A multicomponent intervention to prevent major bleeding complications in older \\ patients receiving warfarin. A randomized, controlled trial. Ann Intern Med 2000 Nov 7;133:687-95.
QUESTION: In older patients requiring warfarin, is multicomponent comprehensive care (MCC) more effective than usual care for reducing major bleeding events?

\section{Design}

Randomised \{allocation concealed*\}†, blinded (data colmonths follow up.

Sources of funding:

National Institute on Aging; Department of Veterans Affairs Health Services Research and Development Service; American Federation for Aging Research; Claude D Pepper Older Americans

Independence Center of Case Western Reserve

University.

For correspondence: Dr R J Beyth, Baylor

College of Medicine

and Houston Center for

Quality of Care and

Utilization Studies,

Houston VAMC (39A),

2002 Holcombe

TX 77030, USA. Fax

$+17135584593$

Multicomponent comprehensive care $(M C C)$ v usual care for patients requiring warfarin $\$$

\begin{tabular}{lllll}
$\begin{array}{l}\text { Outcome at } 6 \\
\text { months }\end{array}$ & MCC & Usual care & RRR $(95 \% \mathrm{Cl})$ & NNT \\
\hline Major bleeding & $4.9 \%$ & $10.5 \%$ & $53.2 \%(-2.7$ to 78.9$)$ & Not significant \\
\hline
\end{tabular}

$\ddagger$ Abbreviations defined in glossary; RRR, NNT, and $\mathrm{Cl}$ calculated from data provided by author.

\section{Setting}

Cuyahoga County, Ohio, USA.

\section{Patients}

325 patients (mean age 75 y, $57 \%$ women) who were $\geqslant$ 65 years of age, received $\geqslant 10000$ units of intravenous unfractionated heparin, resided in the area, and had planned warfarin treatment for $\geqslant 10$ days. Exclusion criteria included treatment with warfarin in the previous 6 months, admission from a nursing home, or enrolment in another clinical trial. Follow up was complete.

\section{Intervention}

163 patients were allocated to MCC, which consisted of a guideline based consultation and patient education, coaching, and self monitoring of prothrombin time with a portable monitor. Home visits were made 3 days after discharge to assess the patients' ability to use the monilectors and outcome assessors),* controlled trial with 6

Boulevard, Houston,

tor. Patients monitored their prothrombin time 3 times during the first week after hospital discharge, once each week for the rest of the first month, and monthly thereafter. 162 patients allocated to usual care were managed by their personal physicians.

\section{COMMENTARY}

The study by Beyth et al has attempted to compare a thromboembolism service and anticoagulation clinic with usual care for managing patients requiring anticoagulation treatment. The study intervention involves both inpatient and outpatient management components but contains elements not yet commonly incorporated into specialty consultation services (eg, a lay educator and point of care INR testing). Not all patients in the MCC group received all components (28\% were self tested), so it is difficult to say which of the components are most important.

Although 6 of the 8 major bleeding events in the MCC group occurred in patients who declined the intervention, 3 of these bleeding events occurred during admission to hospital when patient behaviour and attitudes toward care would have less effect on outcome. Furthermore, almost $50 \%$ of all major bleeding events occurred during the index hospitalisation, not during subsequent outpatient warfarin treatment. Thus, assigning and understanding any effect of the patient's decision to decline the intervention on their bleeding episode would be difficult.

About 20\% of those patients who were offered the intervention declined it. Although this finding may be in keeping with patient participation in other clinical trials, clinicians should remember that not all components of this intervention are applicable to every patient.

The authors did not do a formal cost analysis. The INR monitors are expensive ( $>$ US $\$ 500 ;>C N \$ 800)$ and generally not covered by third party payers. Most of the non-diagnostic components of the intervention programme, however, can and are done by other health professionals, such as pharmacists or nurses, in established thromboembolism services and anticoagulation clinics. The clinical benefits and thus the potential cost benefits of coordinated approaches to anticoagulation management as described in this study await a larger randomised trial. ${ }^{1}$

Intuitively, the components of this intervention are sensible, and certain elements (eg, heparin nomograms, anticoagulation monitoring sheets, and patient education) can be easily adapted to care maps and other standardised approaches to patient management. However, more evidence is needed to determine the best approach for managing patients requiring anticoagulation treatment.

Bill Bartle, PharmD Sunnybrook and Women's College Health Sciences Centre Toronto, Ontario, Canada

1 Ansell J, Hirsh J, Dalen J, et al. Managing oral anticoagulant therapy. Chest 2001;119(suppl 1):S22-38. 